\title{
Comparing the Pre- and Post-specified Peer Models for Key Agreement
}

\author{
Alfred Menezes and Berkant Ustaoglu \\ Department of Combinatorics \& Optimization, University of Waterloo \\ \{ajmeneze, bustaoglu\}@uwaterloo.ca
}

\begin{abstract}
In the pre-specified peer model for key agreement, it is assumed that a party knows the identifier of its intended communicating peer when it commences a protocol run. On the other hand, a party in the post-specified peer model for key agreement does not know the identifier of its communicating peer at the outset, but learns the identifier during the protocol run. In this paper we compare the security assurances provided by the Canetti-Krawczyk security definitions for key agreement in the pre- and post-specified peer models. We give examples of protocols that are secure in one model but insecure in the other. We also enhance the Canetti-Krawczyk security models and definitions to encompass a class of protocols that are executable and secure in both the pre- and post-specified peer models.
\end{abstract}

\section{Introduction}

In 1993, Bellare and Rogaway [1] presented the first formal security model and security definition for key agreement. The model and associated definitions evolved over the years, culminating in the 2001 work of Canetti and Krawczyk [4] and its recent extension by LaMacchia, Lauter and Mityagin [13. In all the aforementioned works, key agreement protocols are analyzed in the so-called pre-specified peer model wherein it is assumed that a party knows the identifier of its intended communicating peer when it commences a run of the protocol. That is, it is assumed that the exchange of identifiers, and possibly also the long-term public keys of the communicating parties, is handled by the application that invokes a run of the protocol.

In 2002, Canetti and Krawczyk [5] introduced the post-specified peer model wherein a party is activated to establish a session key knowing only a destination address (such as the IP address of a server) of the communicating peer, and only learns the peer's identifier during the execution of the protocol. According to [5], this scenario is common in practical settings where the peer's identifier is simply unavailable at the outset, or if one party wishes to conceal its identity from eavesdroppers or active adversaries. The IKE protocols [89] (see also [10]) are important examples of key agreement protocols that provide the option of identity concealment.

In the remainder of this paper we will not consider the identity concealment attribute of key agreement protocols. We will often shorten 'pre-specified peer model' to 'pre model', and 'post-specified peer model' to 'post model'. 
We say that a key agreement protocol designed for one of the pre or post models is executable in the other model if it can be run in the second model without requiring any additional message flows (and without making any fundamental changes to the protocol description). It is clear that any key agreement protocol designed for the post model is executable in the pre model. Indeed, if the peer's identifier (and long-term public key) is not needed at the start of the protocol, then the protocol can also be executed given the peer's identifier. Canetti and Krawczyk observed that the $\Sigma_{0}$ key agreement protocol is secure in the post model with respect to the security definition given in 5], but not secure in the pre model with respect to the security definition given in 4 . Hence, even though any protocol designed for the post model can be executed in the pre model, security in the post model of [5] does not guarantee security in the pre model of [4].

In this paper we explore the executability and security in the post model of key agreement protocols that have been designed for and analyzed in the pre model. Of course any protocol designed for the pre model can be modified for the post model by adding message flows which include the identifiers and long-term public keys of the communicating parties; however such a modification does not conform to our notion of executability because of the additional message flows. We provide an example of a key agreement protocol that is secure in the pre model but is not executable in the post model. We also observe that the HMQV protocol 11, which has been proven to be secure in the pre model, is executable in the post model (without the addition of message flows) but not secure unless additional measures are taken. These examples illustrate the essential differences between the two models, and highlight the danger of running in the post model a protocol that has only been analyzed in the pre model.

It is natural then to ask when a protocol secure in one model is executable and secure in the other model. We identify a class of modifiable key agreement protocols that have been designed for the pre model but can be executed with minimal modifications in the post model. This class includes many of the protocols that have been proposed in the literature including station-to-station 7], UM [19 16], MQV [15], Boyd-Mao-Paterson [2], HMQV [1], KEA+ [14, NAXOS 13. CMQV [20] and Okamoto [18. (See 3] for an extensive list of key establishment protocols.) Such protocols have a hybrid description that combine the specification for the pre model and the specification of the modified protocol suitable for the post model. We develop a combined model and associated security definition that aims to simultaneously capture the security assurances (and more) of the extended Canetti-Krawczyk pre-specified peer model 13 and the CanettiKrawczyk post-specified peer model [5]. The combined model has the feature that if a hybrid key agreement protocol is proven secure in that model, then its specializations are guaranteed to be secure when run in the pre and post models.

The remainder of this paper is organized as follows. In 92 we provide informal overviews of the Canetti-Krawczyk pre and post models and security definitions for key agreement. The differences between the two models are explored in $\$ 3$. Protocol $\mathcal{P}$ is described in 3.1 as an example of a protocol that is secure in the 
pre model but not executable in the post model. In 3.2 we describe an attack on HMQV, demonstrating that the protocol is not secure in the post model. The $\Sigma_{0}$ protocol, which is an example of a protocol that is secure in the post model but insecure in the pre model, is revisited in 3.3. Our combined model and security definition are presented in $\$ 4$ The NAXOS-C protocol is presented in $\$ 5$ as an example of a protocol that is secure in the combined model.

Notation and Terminology. Let $G=\langle g\rangle$ denote a multiplicatively-written cyclic group of prime order $q$, and let $G^{*}=G \backslash\{1\}$. The Computational Diffie-Hellman (CDH) assumption in $G$ is that computing $\operatorname{CDH}(U, V)=g^{u v}$ is infeasible given $U=g^{u}$ and $V=g^{v}$ where $u, v \in_{R}[1, q-1]$. The Decisional Diffie-Hellman (DDH) assumption in $G$ is that distinguishing DH triples $\left(g^{a}, g^{b}, g^{a b}\right)$ from random triples $\left(g^{a}, g^{b}, g^{c}\right)$ is infeasible. The Gap Diffie-Hellman $(G D H)$ assumption in $G$ is that the $\mathrm{CDH}$ assumption holds even when a CDH solver is given a DDH oracle that distinguishes $\mathrm{DH}$ triples from random triples.

We consider Diffie-Hellman type protocols where the two communicating parties exchange static (long-term) and ephemeral (one-time) public keys. Party $\hat{A}$ 's static private key is an integer $a \in_{R}[1, q-1]$, and her corresponding static public key is $A=g^{a}$. Similarly, party $\hat{B}$ has a static key pair $(b, B)$, and so on. A certifying authority (CA) issues certificates that binds a party's identifier to its static public key. We do not assume that the $\mathrm{CA}$ requires parties to prove possession of their static private keys, but we do insist that the CA verifies that static public keys belong to $G^{*}$. We restrict our attention to protocols where a party $\hat{A}$ called the initiator commences the protocol by selecting an ephemeral key pair and then sends the ephemeral public key (and possibly other data) to the second party. In our protocols, the ephemeral private key is either a randomly selected integer $x \in[1, q-1]$ or a randomly selected binary string $\tilde{x}$ which is used together with the static private key to derive an integer $x \in[1, q-1]$, and the corresponding ephemeral public key is $X=g^{x}$. Upon receipt of $X$, the responder $\hat{B}$ selects an ephemeral private key $y$ or $\tilde{y}$ and sends $Y=g^{y}$ (and possibly other data) to $\hat{A}$. The parties may exchange some additional messages, after which they compute a session key. We use $\mathcal{I}$ and $\mathcal{R}$ to denote the constant strings "initiator" and "responder".

\section{Security Definitions for Key Agreement}

We provide overviews of the Canetti-Krawczyk pre- and post-specified peer models for key agreement and the associated security definitions. For full details and further explanations refer to [4] and [5].

\section{$2.1 \quad$ Pre-specified Peer Model}

Communications take place in a multi-party system, where the parties are identified by $\hat{A}, \hat{B}, \hat{C}, \ldots$ At any given point in time, a party may be engaged in multiple instances of the protocol, each called a session. A session is created at $\hat{A}$ via a message containing at least three parameters $(\hat{A}, \hat{B}, s)$, where $\hat{A}$ is the 
session's owner, $\hat{B}$ is the intended peer, and $s$ is a number that is unique among all sessions owned by $\hat{A}$. ( $\hat{A}$ uses $s$ to direct incoming messages to the appropriate session within $\hat{A}$.) Once created, a session is said to be active and maintains a session state where session-specific short-lived data such as an ephemeral private key is stored. The session processes incoming messages and produces outgoing messages. A session may abort without producing a session key, or may complete by accepting a session key and erasing its session state.

The adversary $\mathcal{M}$, modeled as a probabilistic Turing machine, controls all communications between parties as well as the activation of sessions. In order to model the possible leakage of secret information, $\mathcal{M}$ is allowed to issue the following queries to parties:

- SessionStateReveal: $\mathcal{M}$ learns the contents of the session state for a (not yet completed) session of its choosing. The session can no longer be activated and stops producing output.

- Expire: $\mathcal{M}$ directs a completed session to delete its session key.

- SessionKeyReveal: $\mathcal{M}$ learns the session key held by a (completed but unexpired) session of its choosing.

- Corrupt: $\mathcal{M}$ learns all the secret information held by a party of its choosing, including the party's static private key, all session states, and all session keys. The party can no longer be activated and stops producing output.

The adversary's goal is to distinguish a session key from a random key. Obviously the adversary should not be allowed to learn the session key by trivial means, for example by asking for the session key via a SessionKeyReveal query. To this end, a session $(\hat{A}, \hat{B}, s)$ is said to be locally exposed if $\mathcal{M}$ issued a SessionStateReveal or SessionKeyReveal query to that session, or if $\mathcal{M}$ issued a Corrupt query to $\hat{A}$ before the session expired (this includes the case in which $\hat{A}$ is corrupted before the session is created). Moreover, the session $(\hat{B}, \hat{A}, s)$ is defined to be matching to the session $(\hat{A}, \hat{B}, s)$, and $(\hat{A}, \hat{B}, s)$ is said to be unexposed if neither this session nor its matching session are locally exposed. Now, $\mathcal{M}$ selects a session that is completed, unexpired, and unexposed, and issues a special Test query to that session. ( $\mathcal{M}$ is not allowed to issue the Test query more than once.) In response, $\mathcal{M}$ is given with equal probability either the session key held by the test session or a random key. $\mathcal{M}$ can continue to issue queries, however must ensure that the test session remains unexposed. Finally, $\mathcal{M}$ is said to win its distinguishing game (and thereby break the protocol) if it guesses correctly whether the key is random or not with success probability significantly greater than $\frac{1}{2}$. A key agreement protocol is said to be secure (in the pre-specified peer model) if (i) uncorrupted parties who complete matching sessions compute the same session key (except with negligible probability); and (ii) there is no adversary $\mathcal{M}$ who wins the distinguishing game.

\subsection{Post-specified Peer Model}

The Canetti-Krawczyk post-specified peer model and associated security definition [5] are essentially the same as in the pre model, but there are two important differences. 
First, a session at $\hat{A}$ is created via a message containing (at least) three parameters $(\hat{A}, \hat{d}, s)$, where $\hat{d}$ is a destination address to which outgoing messages should be delivered. That is, party $\hat{A}$ does not know the identifier of its peer when it starts the session. During the course of the protocol run, $\hat{A}$ learns the (alleged) identifier $\hat{B}$ of the communicating party; this party is referred to as $\hat{A}$ 's peer for that session.

Second, the definition of a matching session is different. Let $(\hat{A}, s)$ be a session that has completed with peer $\hat{B}$. Then a session $(\hat{B}, s)$ is said to be matching to $(\hat{A}, s)$ if either (i) $(\hat{B}, s)$ has not yet completed; or (ii) $(\hat{B}, s)$ has completed and its peer is $\hat{A}$. Condition (i) is necessary because the incomplete session $(\hat{B}, s)$ may not yet have determined its peer and hence could have been communicating with $(\hat{A}, s)$, in which case exposure of $(\hat{B}, s)$ could possibly reveal non-trivial information about the session key held by $(\hat{A}, s)$.

\section{Differences between the Two Models}

This section presents three examples to illustrate the differences between the Canetti-Krawczyk security definitions for key agreement in the pre- and postspecified peer models. Protocol $\mathcal{P}$ is secure in the pre model, but cannot be executed in the post model. HMQV is an example of a protocol that is secure in the pre model, and executable but not secure in the post model. The $\Sigma_{0}$ protocol is secure in the post model but insecure in the pre model.

\subsection{Protocol $\mathcal{P}$}

We present a two-pass Diffie-Hellman key agreement protocol $\mathcal{P}$. The protocol can be proven secure in the pre-specified peer model under the GDH assumption and where $\mathrm{H}$ and $\mathrm{H}_{2}$ are modeled as random functions. (The reductionist security argument is elementary but tedious, and hence is omitted.) Observe that the initiator $\hat{A}$ cannot prepare the first outgoing message without knowledge of the peer's identifier $\hat{B}$ and static public key $B$. Hence, unless protocol $\mathcal{P}$ is modified in a fundamental way, it cannot be executed in the post-specified peer model without additional message flows to exchange identifiers and static public keys.

1. On input $(\hat{A}, \hat{B}, s)$, party $\hat{A}$ (the initiator) does the following:
(a) Create an active session $(\hat{A}, \hat{B}, s, \mathcal{I})$.
(b) Select an ephemeral private key $x \in_{R}[1, q-1]$.
(c) Compute $X=g^{x}$ and $t_{A}=H_{2}\left(B^{a}, \mathcal{I}, s, \hat{A}, \hat{B}, X\right)$.
(d) Send $\left(\hat{B}, \hat{A}, s, X, t_{A}\right)$ to $\hat{B}$.

2. Upon receiving $\left(\hat{B}, \hat{A}, s, X, t_{A}\right)$, party $\hat{B}$ (the responder) does the following:
(a) Create an active session $(\hat{B}, \hat{A}, s, \mathcal{R})$.
(b) Verify that $X \in G^{*}$.
(c) Compute $\sigma_{s}=A^{b}$ and verify that $t_{A}=H_{2}\left(\sigma_{s}, \mathcal{I}, s, \hat{A}, \hat{B}, X\right)$.
(d) Select an ephemeral private key $y \in_{R}[1, q-1]$.
(e) Compute $Y=g^{y}, t_{B}=H_{2}\left(\sigma_{s}, \mathcal{R}, s, \hat{B}, \hat{A}, Y\right)$, and $k=H\left(X^{y}, X, Y\right)$.
(f) Destroy $y$ and $\sigma_{s}$. 
(g) Send $\left(\hat{A}, \hat{B}, s, \mathcal{I}, Y, t_{B}\right)$ to $\hat{A}$.

(h) Complete the session $(\hat{B}, \hat{A}, s, \mathcal{R})$ and accept $k$ as the session key.

3. Upon receiving $\left(\hat{A}, \hat{B}, s, \mathcal{I}, Y, t_{B}\right)$, party $\hat{A}$ checks that she owns an active session with identifier $(\hat{A}, \hat{B}, s, \mathcal{I})$. If so, then $\hat{A}$ does the following:

(a) Verify that $Y \in G^{*}$.

(b) Verify that $t_{B}=H_{2}\left(B^{a}, \mathcal{R}, s, \hat{B}, \hat{A}, Y\right)$.

(c) Compute $k=H\left(Y^{x}, X, Y\right)$.

(d) Destroy $x$.

(e) Complete the session $(\hat{A}, \hat{B}, s, \mathcal{I})$ by accepting $k$ as the session key.

\subsection{HMQV Protocol}

HMQV 11] is an efficient two-pass Diffie-Hellman key agreement protocol that has been proven to be secure in the pre-specified peer model under the $\mathrm{CDH}$ and KEA1 assumptions and where the hash functions employed are modeled as random functions 11 The following informal description of the protocol omits some technical details that are not relevant to our analysis 2

Let $\bar{H}$ denote a hash function whose outputs are bitstrings of length $l$, where $l$ is half the bitlength of the group order $q$. In HMQV, the initiator $\hat{A}$ sends $(\hat{B}, \hat{A}, X)$ to $\hat{B}$, who responds with $(\hat{A}, \hat{B}, Y)$. Party $\hat{A}$ computes the session key $k=H\left(\sigma_{A}\right)$, where $\sigma_{A}=\left(Y B^{e}\right)^{x+d a}$ and $d=\bar{H}(X, \hat{B})$ and $e=\bar{H}(Y, \hat{A})$. Party $\hat{B}$ computes the same session key as $k=H\left(\sigma_{B}\right)$, where $\sigma_{B}=\left(X A^{d}\right)^{y+e b}$.

Unlike protocol $\mathcal{P}$, HMQV is executable in the post-specified peer model. Indeed, the initiator can prepare the first message (which essentially consists of the ephemeral public key $X$ ) without knowledge of the peer's identifier $\hat{B}$ or static public key $B$. It is natural then to ask whether HMQV is secure in the post model. This is also important because the version of HMQV that is being considered for standardization by the P1363 working group [12] does not mandate that the protocol be executed in the pre model (i.e., there is no requirement that the communicating parties possess each other's identifiers and static public keys prior to a protocol run), and consequently the protocol may in fact be executed in the post model in applications where the responder's identifier is not available to the initiator at the beginning of the protocol run.

We describe an attack which demonstrates that HMQV (without further modification such as the addition of message flows to exchange identifiers and static public keys) is not secure in the post model. The attack makes the following plausible assumptions: (i) the group order $q$ is a 160-bit prime and so the outputs of $\bar{H}$ have bitlength 80; (ii) the best attack on the $\mathrm{CDH}$ problem in $G$ takes approximately $2^{80}$ steps; (iii) there are at least $2^{20}$ honest (i.e., uncorrupted) parties; (iv) a party can select its own identifier; and (v) the certification authority does

\footnotetext{
${ }^{1}$ The security definition used in 11 is stronger than the security definition outlined in 2.1 in the sense that the adversary is granted certain additional capabilities. For example, the adversary is allowed to register a static key pair at any time thus allowing the modeling of attacks by malicious insiders.

${ }^{2}$ In particular, we omit session identifiers and assume that all static and ephemeral public key are fully validated, i.e., verified as belonging to $G^{*}$.
} 
not require parties to prove knowledge of the static private keys corresponding to their static public keys during registration 3 The attack proceeds as follows.

1. The adversary $\mathcal{M}$ induces $\hat{A}$ to create a session with a destination address $\hat{d}$ controlled by $\mathcal{M}$. In response, $\hat{A}$ selects ephemeral key pair $(x, X)$ and sends $(\hat{d}, \hat{A}, X)$.

2. $\mathcal{M}$ intercepts $(\hat{d}, \hat{A}, X)$ and does the following:

(a) Compute $S=\{(\hat{C}, \bar{H}(X, \hat{C})) \mid \hat{C}$ is an honest party $\}$.

(b) Select an identifier $\hat{M}$ (not the same as the identifier of an honest party) such that $(\hat{B}, \bar{H}(X, \hat{M})) \in S$ for some $\hat{B}$.

(c) Select $M=B$ as $\hat{M}$ 's static public key (note that $\mathcal{M}$ does not know the corresponding private key).

(d) Send $(\hat{B}, \hat{A}, X)$ to $\hat{B}$.

3. $\mathcal{M}$ intercepts $\hat{B}$ 's reply $(\hat{A}, \hat{B}, Y)$ and sends $(\hat{A}, \hat{M}, Y)$ to $\hat{A}$.

Party $\hat{A}$ computes the session key $k=H\left(\sigma_{A}\right)$, where $\sigma_{A}=\left(Y M^{e}\right)^{x+d a}$ and $d=\bar{H}(X, \hat{M})$ and $e=\bar{H}(Y, \hat{A})$. Party $\hat{B}$ computes the session key $k^{\prime}=H\left(\sigma_{B}\right)$, where $\sigma_{B}=\left(X A^{d^{\prime}}\right)^{y+e^{\prime} b}$ and $d^{\prime}=\bar{H}(X, \hat{B})$ and $e^{\prime}=\bar{H}(Y, \hat{A})$. Since $d^{\prime}=d$, $e^{\prime}=e$, and $M=B$, we have $\sigma_{A}=\sigma_{B}$ and hence $k^{\prime}=k$. The problem is that while $\hat{B}$ correctly believes that $k$ is shared with $\hat{A}$, party $\hat{A}$ mistakenly believes that $k$ is shared with $\hat{M}$. Thus $\mathcal{M}$ has successfully launched an 'unknown keyshare' or 'identity misbinding' attack on HMQV in the post model. The expected running time of the attack is about $2^{60}$ (for step $2 \mathrm{~b}$ ). Since most of the work has to be done online, the attack cannot be considered practical. Nevertheless it demonstrates that HMQV does not attain an 80-bit security level in the post model as it presumably does in the pre model.

The mechanisms of the attack were outlined in Remark 7.2 of [11. However, the adversary considered in 11. operates in a different setting, namely the pre model where party $\hat{A}$ precomputes and stores her ephemeral public keys $X$ which are then inadvertently leaked to $\mathcal{M}$ before $\hat{A}$ uses them in a session. Three countermeasures were proposed in [11] for foiling this attack: (i) increase the output length of $\bar{H}$ to 160 bits; (ii) include the identifiers $\hat{A}, \hat{B}$ in the key derivation function whereby the session key is computed as $k=H(\sigma, \hat{A}, \hat{B})$; and (iii) include random nonces (which are not precomputed and stored) in the derivation of exponents $d$ and $e$, whereby the exponents are computed as $d=\bar{H}\left(X, \hat{B}, \nu_{A}\right)$ and $e=\bar{H}\left(Y, \hat{A}, \nu_{B}\right)$ where $\nu_{A}$ and $\nu_{B}$ are $\hat{A}$ 's and $\hat{B}$ 's nonces, respectively. Countermeasures (i) and (ii) are successful in thwarting the attack described above on HMQV in the post model. However, it can easily be seen that countermeasure (iii) does not prevent the attack in the post model, thus demonstrating that the two attacks are indeed different. The reason countermeasure (iii) fails is that, unlike in the pre model, the peer's identifier is not known to $\hat{A}$ when she creates the session in the post model.

${ }^{3}$ In $[1]$ it is noted that the HMQV security proof does not depend on the CA performing any proof-of-possession checks. 


\section{$3.3 \quad \Sigma_{0}$ Protocol}

The $\Sigma_{0}$ protocol [5] is a simplified version of one of the IKE key agreement protocols. In the protocol description below, PRF is a pseudorandom function family, MAC is a message authentication code algorithm, and $\operatorname{sig}_{A}$ and $\operatorname{sig}_{B}$ are the signing algorithms for $\hat{A}$ and $\hat{B}$, respectively.

1. Party $\hat{A}$ (the initiator) selects an ephemeral key pair $(x, X)$, initializes the session identifier to $(\hat{A}, s)$, and sends $\left(\hat{d}_{B}, \hat{d}_{A}, s, X\right)$. Here $\hat{d}_{A}$ and $\hat{d}_{B}$ are destination addresses for $\hat{A}$ and $\hat{B}$, respectively.

2. Upon receipt of $\left(\hat{d}_{B}, \hat{d}_{A}, s, X\right), \hat{B}$ (the responder) selects an ephemeral key pair $(y, Y)$, and computes $\sigma=X^{y}, k=\operatorname{PRF}_{\sigma}(0)$, and $k^{\prime}=\operatorname{PRF}_{\sigma}(1) . \hat{B}$ then destroys $y$ and $\sigma$, initializes the session identifier to $(\hat{B}, s)$, and sends $m_{1}=\left(\hat{d}_{A}, \hat{B}, s, Y, \operatorname{sig}_{B}(\mathcal{R}, s, X, Y), \operatorname{MAC}_{k^{\prime}}(\mathcal{R}, s, \hat{B})\right)$.

3. Upon receiving $m_{1}, \hat{A}$ computes $\sigma=Y^{x}, k=\operatorname{PRF}_{\sigma}(0)$, and $k^{\prime}=\operatorname{PRF}_{\sigma}(1)$. $\hat{A}$ then verifies the signature and MAC tag in $m_{1}$, and sends $m_{2}=(\hat{B}, \hat{A}, s$, $\left.\operatorname{sig}_{A}(\mathcal{I}, s, Y, X), \operatorname{MAC}_{k^{\prime}}(\mathcal{I}, s, \hat{A})\right)$. Finally, $\hat{A}$ accepts the session key $k$ with peer $\hat{B}$, and erases the session state.

4. Upon receiving $m_{2}, \hat{B}$ verifies the signature and MAC tag in $m_{2}$, accepts the session $k$ with peer $\hat{A}$, and erases the session state.

In [5], the $\Sigma_{0}$ protocol is proven secure in the post-specified peer model provided that the $\mathrm{DDH}$ assumption holds in $G$ and that the PRF, MAC, and sig primitives are secure. However, the following attack described in [5] shows that $\Sigma_{0}$ is not secure in the pre-specified peer model.

1. Create a session $(\hat{A}, \hat{B}, s)$ at $\hat{A}$.

2. Intercept $\hat{A}$ 's outgoing message $(\hat{B}, \hat{A}, s, X)$ and send $(\hat{B}, \hat{M}, s, X)$ to $\hat{B}$.

3. Intercept $\hat{B}$ 's response $\left(\hat{M}, \hat{B}, s, Y, S_{B}, t_{B}\right)$, where $S_{B}=\operatorname{sig}_{B}(\mathcal{R}, s, X, Y)$ and $t_{B}=\operatorname{MAC}_{k^{\prime}}(\mathcal{R}, s, \hat{B})$, and send $\left(\hat{A}, \hat{B}, s, Y, S_{B}, t_{B}\right)$ to $\hat{A}$.

4. The session $(\hat{A}, \hat{B}, s)$ at $\hat{A}$ completes and accepts $k$ as the session key.

5. Intercept and delete $\hat{A}$ 's final message, and issue a SessionStateReveal query to the session $(\hat{B}, \hat{M}, s)$ thus learning $k$ and $k^{\prime}$.

6 . Issue the Test query to the session $(\hat{A}, \hat{B}, s)$ and use knowledge of $k$ to win the distinguishing game.

Notice that the attack is legitimate in the pre-specified peer model since the exposed session $(\hat{B}, \hat{M}, s)$ is not matching to the test session $(\hat{A}, \hat{B}, s)$. On the other hand, such an attack is not permitted in the post-specified peer model because in step 5 of the attack the session $(\hat{B}, s)$ is still incomplete and therefore matching to the Test session (and thus cannot be exposed). This is all rather counterintuitive since one would expect that if a protocol is secure when the initiator does not have a priori knowledge of the peer's identifier, then it should remain secure when the peer's identifier is known at the outset.

One feature of both the pre and post models is that an exposed session does not produce any further output. In practice, however, one might desire the assurance that a particular session is secure even if the adversary learns some secret state information (such as an ephemeral private key) associated with that 
session or its matching session. For this reason, the security models in recent papers such as [1], [13] and 20] permit exposed sessions to continue producing output, and furthermore allow the adversary to issue a SessionStateReveal query (or its equivalent) to the Test session and its matching session (cf. \$4.3). However, if the adversary $\mathcal{M}$ were equipped with these extra capabilities, then the $\Sigma_{0}$ protocol would be insecure in both the pre and post models since $\mathcal{M}$ could issue a SessionStateReveal query to $(\hat{A}, s)$ after step 1 to learn $x$ and thereafter compute the session key. Furthermore, the $\Sigma_{0}$ protocol falls in the post model to the following analogue of the attack described above. The attack is a little more realistic than the attack described above in the pre model because we now assume that the SessionStateReveal query does not yield the session key $k$ (which may be stored in secure memory). $\mathcal{M}$ 's actions are the following:

1. Create a session $(\hat{A}, s)$ at $\hat{A}$ with peer destination address $\hat{d}_{B}$.

2. Intercept $\hat{A}$ 's outgoing message $\left(\hat{d}_{B}, \hat{d}_{A}, s, X\right)$ and send $\left(\hat{d}_{B}, \hat{d}_{M}, s, X\right)$ to $\hat{B}$.

3. Intercept $\hat{B}$ 's response $\left(\hat{d}_{M}, \hat{B}, s, Y, S_{B}, t_{B}\right)$, where $S_{B}=\operatorname{sig}_{B}(\mathcal{R}, s, X, Y)$ and $t_{B}=\operatorname{MAC}_{k^{\prime}}(\mathcal{R}, s, \hat{B})$, and send $\left(\hat{d}_{A}, \hat{B}, s, Y, S_{B}, t_{B}\right)$ to $\hat{A}$.

4. Intercept $\hat{A}$ 's final message and delete it. The session $(\hat{A}, s)$ completes with peer $\hat{B}$ and session key $k$.

5. Issue a SessionStateReveal query to the incomplete session $(\hat{B}, s)$ and learn the MAC key $k^{\prime}$.

6. Compute $S_{M}=\operatorname{sig}_{M}(\mathcal{I}, s, Y, X)$ and $t_{M}=\operatorname{MAC}_{k^{\prime}}(\mathcal{I}, s, \hat{M})$, and send $\left(\hat{B}, \hat{M}, s, S_{M}, t_{M}\right)$ to $\hat{B}$.

The session $(\hat{B}, s)$ completes with peer $\hat{M}$ and session key $k$. Thus $\mathcal{M}$ has successfully launched an unknown key-share attack on $\Sigma_{0}$ in the post model. The two attacks demonstrate that a protocol proven secure in the post-specified peer model of [5] may no longer be secure if exposed sessions are allowed to continue producing output.

\section{Combining the Two Models}

In this section we introduce the notion of a modifiable key agreement protocol - protocols designed for the pre-specified peer model but which can be adapted with minor changes to be executable in the post-specified peer model. We also introduce the notion of a hybrid key agreement protocol, which simultaneously describes a modifiable protocol and its modification suitable for the post model. We then develop a security definition that, if satisfied by a hybrid protocol, guarantees that the associated protocols are secure in the pre and post models.

\subsection{Modifiable Protocols}

Consider a key agreement protocol $\Pi$ designed for the pre model where the first outgoing message prepared by the initiator $\hat{A}$ is of the form ( $\hat{B}, \hat{A}$, RoundOne $)$. Then $\Pi$ is said to be modifiable if RoundOne can be computed before the session 
is created at $\hat{A}$; in particular, this means that RoundOne does not depend on $\hat{B}$ 's identifier or static public key.

A modifiable protocol $\Pi$ can be easily adapted for the post-specified peer model by incorporating identity establishment into the protocol flows. The required changes are the following. The initiator $\hat{A}$, who is activated to create a session with a destination address $\hat{d}$ (and without knowledge of the recipient's identifier or static public key), sends ( $\hat{d}, \hat{A}$, RoundOne) as her first outgoing message. Since this message contains the identifier $\hat{A}$, the responder has all the information he needs to prepare his first outgoing message as specified by $\Pi$. The responder appends his identifier to this outgoing message (if the message does not already contain the identifier). After $\hat{A}$ receives this reply, both $\hat{A}$ and the responder can proceed with $\Pi$ without any further modifications. Notice that the modified protocol $\Pi^{\prime}$ has the same number of message flows as the original protocol $\Pi$; except for appending a public value to the first outgoing message, the remainder of the protocol remains the same.

As mentioned in \$1 the class of modifiable key agreement protocols includes many of the protocols that have been proposed in the literature. However, not all key agreement protocols are modifiable; for example, protocol $\mathcal{P}$ defined in 3.1 is not modifiable. Furthermore, as demonstrated by the attack on HMQV in 3.2 security of a modifiable protocol $\Pi$ in the pre model does not imply security of the modified protocol $\Pi^{\prime}$ in the post model.

\subsection{Hybrid Protocols}

Suppose that $\Pi$ is a modifiable key agreement protocol, and $\Pi^{\prime}$ its modification suitable for the post model. The specification of $\Pi$ and $\Pi^{\prime}$ can be combined as described below, resulting in a protocol $\tilde{\Pi}$ called a hybrid protocol.

We use $\tilde{A}$ to denote either an identifier $\hat{A}$ or a destination address $\hat{d}$ that can be used to send messages to some party $\hat{A}$ whose identifier is not known to the sender; note that the address $\hat{d}$ may not necessarily be under $\hat{A}$ 's control. In the description of $\tilde{\Pi}$, a session is created at initiator $\hat{A}$ via a message containing $(\hat{A}, \tilde{B})$. The first outgoing message from $\hat{A}$ is $(\tilde{B}, \hat{A}$, RoundOne). The responder $\hat{B}$ includes the identifiers $\hat{A}$ and $\hat{B}$ in his response, and the remainder of the protocol description is the same as for $\Pi$.

A hybrid protocol $\tilde{\Pi}$ can be specialized for the pre model by using an identifier $\hat{B}$ for $\tilde{B}$. Protocol $\tilde{\Pi}$ can also be specialized for the post model by using a destination address for $\tilde{B}$. An example of a hybrid protocol is given in $\$ 5$.

\subsection{Combined Security Model}

This section describes a "combined" model and associated security definition that aims to simultaneously capture the security assurances of the pre- and post-specified peer models. That is, if a hybrid protocol $\tilde{\Pi}$ is proven secure with respect to the new definition, then its specializations $\Pi$ and $\Pi^{\prime}$ are guaranteed to be secure when run in the pre and post models, respectively. More precisely, when run in the pre model, $\Pi$ satisfies the extended Canetti-Krawczyk (eCK) definition [13] suitably enhanced to capture attacks where an adversary is able to 
learn ephemeral public keys of parties before they are actually used in a protocol session 4 Such attacks were considered by Krawczyk [11, but were not incorporated into his security model. When run in the post model, the modified protocol $\Pi \Pi^{\prime}$ satisfies a strengthened version of the Canetti-Krawczyk definition from [5], suitably enhanced to offer security assurances similar to the eCK definition (including resistance to attacks where the adversary learns ephemeral private keys of the session being attacked) and to capture attacks where the adversary learns ephemeral public keys before they are actually used.

Instead of using pre-determined session numbers $s$ to identify sessions (cf. 2.1), our session identifiers will consist of the identities of the communicating parties together with a concatenation of the messages exchanged during a protocol run. As shown in [6], this notion of session identifier yields a security model for key agreement that is at least as strong as other security models.

Notation. We assume that messages are represented as binary strings. If $m$ is a vector then \# $m$ denotes the number of its components. We say that two vectors $m_{1}$ and $m_{2}$ are matched, written $m_{1} \sim m_{2}$, if the first $t=\min \left\{\# m_{1}, \# m_{2}\right\}$ components of the vectors are pairwise equal as binary strings. We write $\hat{A} \equiv \widetilde{D}$ if either $\tilde{D}=\hat{A}$ or if $\tilde{D}$ is a destination address that can be used to send messages to $\hat{A}$.

Session Creation. A party $\hat{A}$ can be activated via an incoming message to create a session. The incoming message has one of the following forms: (i) $(\hat{A}, \tilde{B})$ or (ii) $(\tilde{A}, \hat{B}, I n)$. If $\hat{A}$ was activated with $(\hat{A}, \tilde{B})$ then $\hat{A}$ is the session initiator; otherwise $\hat{A}$ is the session responder.

Session Initiator. If $\hat{A}$ is the session initiator then $\hat{A}$ creates a separate session state where session-specific short-lived data is stored, and prepares a reply Out that includes an ephemeral public key $X$. The session is labeled active and identified via a (temporary and incomplete) session identifier $s=(\hat{A}, \tilde{B}, \mathcal{I}$, Comm $)$ where Comm is initialized to Out. The outgoing message is ( $\tilde{B}, \hat{A}$, Out).

Session Responder. If $\hat{A}$ is the session responder then $\hat{A}$ creates a separate session state and prepares a reply Out that includes an ephemeral public key $X$. The session is labeled active and identified via a (temporary and incomplete) session identifier $s=(\hat{A}, \hat{B}, \mathcal{R}$, Comm) where Comm $=($ In, Out $)$. The outgoing message is $(\hat{B}, \hat{A}, \mathcal{I}$, In, Out).

Session Update. A party $\hat{A}$ can be activated to update a session via an incoming message of the form $(\hat{A}, \hat{B}$, role, Comm, In $)$, where role $\in\{\mathcal{I}, \mathcal{R}\}$. Upon receipt of this message, $\hat{A}$ checks that she owns an active session with identifier $s=$ $(\hat{A}, \hat{B}$, role, Comm $)$ or $s=(\hat{A}, \hat{d}$, role, Comm $)$ where $\hat{d}$ is a destination address; except with negligible probability, $\hat{A}$ can own at most one such session. If no such session exists then the message is rejected. If a session $s=(\hat{A}, \hat{d}$, role, Comm $)$ or $s=(\hat{A}, \hat{B}$, role, Comm) exists, then in the former case $\hat{A}$ updates the session

\footnotetext{
${ }^{4}$ As discussed in [11, such attacks may be possible in situations where a party pre-
} computes ephemeral public keys in order to improve on-line performance. 
identifier to $s=(\hat{A}, \hat{B}$, role, Comm $)$; in either case, $\hat{A}$ updates $s$ by appending In to Comm. If the protocol requires a response by $\hat{A}$, then $\hat{A}$ prepares the required response Out; the outgoing message is ( $\hat{B}, \hat{A}$, role, Comm, Out) where role is $\hat{B}$ 's role as perceived by $\hat{A}$, and the session identifier is updated by appending Out to Comm. If the protocol specifies that no further messages will be received, then the session completes and accepts a session key.

Matching Sessions. Since ephemeral public keys are selected at random on a per-session basis, session identifiers are unique except with negligible probability. Party $\hat{A}$ is said to be the owner of a session $(\hat{A}, \tilde{B}, *, *)$. For a session $(\hat{A}, \hat{B}, *, *)$ we call $\hat{B}$ the session peer; together $\hat{A}$ and $\hat{B}$ are referred to as the communicating parties. Let $s=\left(\hat{A}, \tilde{B}\right.$, role $\left._{A}, \mathrm{Comm}_{A}\right)$ be a session owned by $\hat{A}$, where role $A \in$ $\{\mathcal{I}, \mathcal{R}\}$. A session $s^{*}=\left(\hat{C}, \tilde{D}\right.$, role $\left._{C}, \operatorname{Comm}_{C}\right)$, where role $C \in\{\mathcal{I}, \mathcal{R}\}$, is said to be matching to $s$ if $\hat{C} \equiv \tilde{B}, \hat{A} \equiv \tilde{D}$, role $_{A} \neq$ role $_{C}$, and Comm $_{C} \sim \mathrm{Comm}_{A}$. It can be seen that the session $s$, except with negligible probability, can have more than one matching session if and only if $\mathrm{Comm}_{A}$ has exactly one component, i.e., is comprised of a single outgoing message.

Aborted Sessions. A protocol may require parties to perform some checks on incoming messages. For example, a party may be required to perform some form of public key validation or verify a signature. If a party is activated to create a session with an incoming message that does not meet the protocol specifications, then that message is rejected and no session is created. If a party is activated to update an active session with an incoming message that does not meet the protocol specifications, then the party deletes all information specific to that session (including the session state and the session key if it has been computed) and aborts the session; such an abortion occurs before the session identifier can be updated. At any point in time a session is in exactly one of the following states: active, completed, aborted.

Adversary. The adversary $\mathcal{M}$ is modeled as a probabilistic Turing machine and controls all communications. In particular, this means that $\hat{A} \equiv \hat{d}$ for all parties $\hat{A}$ and all destination addresses $\hat{d}$. Parties submit outgoing messages to $\mathcal{M}$, who makes decisions about their delivery. The adversary presents parties with incoming messages via Send(message), thereby controlling the activation of parties. The adversary does not have immediate access to a party's private information, however in order to capture possible leakage of private information $\mathcal{M}$ is allowed to make the following queries:

- StaticKeyReveal $(\hat{A}): \mathcal{M}$ obtains $\hat{A}$ 's static private key.

- EphemeralKeyReveal $(s): \mathcal{M}$ obtains the ephemeral private key held by session $s: 5$ We will henceforth assume that $\mathcal{M}$ issues this query only to sessions that hold an ephemeral private key.

${ }^{5}$ The EphemeralKeyReveal query can be made functionally equivalent to the SessionStateReveal query by defining the ephemeral private key to consist of all ephemeral secret data that a session may hold. 
- SessionKeyReveal( $(s)$ : If $s$ has completed then $\mathcal{M}$ obtains the session key held by $s$. We will henceforth assume that $\mathcal{M}$ issues this query only to sessions that have completed.

- EphemeralPublicKeyReveal $(\hat{A}): \mathcal{M}$ obtains the ephemeral public key that $\hat{A}$ will use the next time a session is created within $\hat{A}$.

- EstablishParty $(\hat{A}, A)$ : This query allows $\mathcal{M}$ to register an identifier $\hat{A}$ and a static public key $A$ on behalf of a party. The adversary totally controls that party, thus permitting the modeling of attacks by malicious insiders. Parties that were established by $\mathcal{M}$ using EstablishParty are called corrupted or adversary controlled. If a party is not corrupted it is said to be honest.

Adversary Goal. To capture the indistinguishability requirement, $\mathcal{M}$ is allowed to make a special query Test $(s)$ to a 'fresh' session $s$. In response, $\mathcal{M}$ is given with equal probability either the session key held by $s$ or a random key. $\mathcal{M}$ meets its goal if it guesses correctly whether the key is random or not. Note that $\mathcal{M}$ can continue interacting with the parties after issuing the Test query, but must ensure that the test session remains fresh throughout $\mathcal{M}$ 's experiment.

Definition 1. Let $s$ be the identifier of a completed session, owned by an honest party $\hat{A}$ with peer $\hat{B}$, who is also honest. Let $s^{*}$ be the identifier of the matching session of $s$, if it exists. Define $s$ to be fresh if none of the following conditions hold:

1. $\mathcal{M}$ issued SessionKeyReveal( $s$ ) or SessionKeyReveal $\left(s^{*}\right)$ (if $s^{*}$ exists).

2. $s^{*}$ exists and $\mathcal{M}$ issued one of the following:

(a) Both StaticKeyReveal $(\hat{A})$ and EphemeralKeyReveal $(s)$.

(b) Both StaticKeyReveal $(\hat{B})$ and EphemeralKeyReveal $\left(s^{*}\right)$.

3. $s^{*}$ does not exist and $\mathcal{M}$ issued one of the following:

(a) Both StaticKeyReveal $(\hat{A})$ and EphemeralKeyReveal $(s)$.

(b) StaticKeyReveal $(\hat{B})$.

Definition 2. A key agreement protocol is secure if the following conditions hold:

1. If two honest parties complete matching sessions then, except with negligible probability, they both compute the same session key.

2. No polynomially bounded adversary $\mathcal{M}$ can distinguish the session key of a fresh session from a randomly chosen session key, with probability greater than $\frac{1}{2}$ plus a negligible fraction.

\section{NAXOS-C Protocol}

In this section we present the hybrid version of the NAXOS-C key agreement protocol, which is essentially the NAXOS protocol of 13] augmented with key confirmation. In the protocol description, $\lambda$ is the security parameters, and $H$ : $\{0,1\}^{*} \rightarrow\{0,1\}^{\lambda} \times\{0,1\}^{\lambda}, H_{1}:\{0,1\}^{*} \rightarrow[1, q-1]$, and $H_{2}:\{0,1\}^{*} \rightarrow\{0,1\}^{\lambda}$ are hash functions. NAXOS-C can be specialized to run in either the pre or the 
post model. Moreover, it can be proven secure in the combined model of 4.3 provided that the GDH assumption holds in $G$ and that the hash functions $H$, $H_{1}$ and $H_{2}$ are modeled as random functions; a reductionist security argument can be found in the full version of this paper [17. Hence NAXOS-C is secure in both the pre- and post-specified peer models.

The purpose of presenting the NAXOS-C protocol is to demonstrate that the security definition of $₫ 4.3$ is useful (and not too restrictive) in the sense that there exist practical protocols that meet the definition under reasonable assumptions. The protocol was designed to allow a straightforward (albeit tedious) reductionist security argument, and has not been optimized. In particular, not all the inputs to the hash functions $H, H_{1}$ and $H_{2}$ may be necessary for security, and in practice $\mathrm{H}_{2}$ would be implemented as a MAC algorithm (with secret key $k^{\prime}$ ).

1. Party $\hat{A}$ (the initiator) does the following:
(a) Select an ephemeral private key $\tilde{x} \in_{R}\{0,1\}^{\lambda}$, and compute $x=H_{1}(a, \tilde{x})$ and $X=g^{x}$.
(b) Destroy $x$.
(c) Initialize the session identifier to $(\hat{A}, \tilde{B}, \mathcal{I}, X)$.
(d) Send $(\tilde{B}, \hat{A}, X)$ to $\tilde{B}$.

2. Upon receiving $(\tilde{B}, \hat{A}, X)$, party $\hat{B}$ (the responder) does the following:

(a) Verify that $X \in G^{*}$.

(b) Select an ephemeral private key $\tilde{y} \in_{R}\{0,1\}^{\lambda}$, and compute $y=H_{1}(b, \tilde{y})$ and $Y=g^{y}$.

(c) Compute $\sigma_{1}=A^{y}, \sigma_{2}=X^{b}$ and $\sigma_{e}=X^{y}$.

(d) Compute $\left(k, k^{\prime}\right)=H\left(\hat{A}, \hat{B}, X, Y, \sigma_{1}, \sigma_{2}, \sigma_{e}\right)$ and $t_{B}=H_{2}\left(k^{\prime}, \mathcal{R}, \hat{B}, \hat{A}, Y, X\right)$.

(e) Destroy $\tilde{y}, y, \sigma_{1}, \sigma_{2}$ and $\sigma_{e}$.

(f) Initialize the session identifier to $\left(\hat{B}, \hat{A}, \mathcal{R}, X, Y, t_{B}\right)$.

(g) Send $\left(\hat{A}, \hat{B}, X, Y, t_{B}\right)$ to $\hat{A}$.

3. Upon receiving $\left(\hat{A}, \hat{B}, X, Y, t_{B}\right)$, party $\hat{A}$ checks that she owns an active session with identifier $(\hat{A}, \tilde{B}, \mathcal{I}, X)$. If so, then $\hat{A}$ does the following:
(a) Verify that $Y \in G^{*}$.
(b) Compute $x=H_{1}(a, \tilde{x}), \sigma_{1}=Y^{a}, \sigma_{2}=B^{x}$ and $\sigma_{e}=Y^{x}$.
(c) Compute $\left(k, k^{\prime}\right)=H\left(\hat{A}, \hat{B}, X, Y, \sigma_{1}, \sigma_{2}, \sigma_{e}\right)$.
(d) Destroy $\tilde{x}, x, \sigma_{1}, \sigma_{2}$ and $\sigma_{e}$.
(e) Verify that $t_{B}=H_{2}\left(k^{\prime}, \mathcal{R}, \hat{B}, \hat{A}, Y, X\right)$.
(f) Compute $t_{A}=H_{2}\left(k^{\prime}, \mathcal{I}, \hat{A}, \hat{B}, X, Y\right)$.
(g) Destroy $k^{\prime}$.
(h) Send $\left(\hat{B}, \hat{A}, X, Y, t_{B}, t_{A}\right)$ to $\hat{B}$.
(i) Update the session identifier to $\left(\hat{A}, \hat{B}, \mathcal{I}, X, Y, t_{B}, t_{A}\right)$ and complete the session by accepting $k$ as the session key.

4. Upon receiving $\left(\hat{B}, \hat{A}, X, Y, t_{B}, t_{A}\right)$, party $\hat{B}$ checks that he owns an active session with identifier $\left(\hat{B}, \hat{A}, \mathcal{R}, X, Y, t_{B}\right)$. If so, then $\hat{B}$ does the following:

(a) Verify that $t_{A}=H_{2}\left(k^{\prime}, \mathcal{I}, \hat{A}, \hat{B}, X, Y\right)$.

(b) Destroy $k^{\prime}$.

(c) Update the session identifier to $\left(\hat{B}, \hat{A}, \mathcal{R}, X, Y, t_{B}, t_{A}\right)$ and complete the session by accepting $k$ as the session key. 


\section{Conclusions}

We compared the Canetti-Krawczyk pre- and post-specified peer models for key agreement, and demonstrated that security in one model does not guarantee security or even executability in the other model. We also presented a combined security model and definition that simultaneously encompasses strengthened versions of the Canetti-Krawczyk definitions. The new definition is stronger in that it permits the adversary to learn ephemeral public keys before they are used, and to learn secret information from the session being attacked. Useful directions for future research would be the development of an optimized protocol that satisfies the new security definition, perhaps modified to allow for identity concealment, and the extension of the definition to capture a wider class of key agreement protocols.

\section{References}

1. Bellare, M., Rogaway, P.: Entity authentication and key distribution. In: Stinson, D.R. (ed.) CRYPTO 1993. LNCS, vol. 773, pp. 232-249. Springer, Heidelberg (1994), http://www.cs.ucdavis.edu/ rogaway/papers/eakd-abstract.html

2. Boyd, C., Mao, W., Paterson, K.: Key agreement using statically keyed authenticators. In: Jakobsson, M., Yung, M., Zhou, J. (eds.) ACNS 2004. LNCS, vol. 3089, pp. 248-262. Springer, Heidelberg (2004)

3. Boyd, C., Mathuria, A.: Protocols for Authentication and Key Establishment. Springer, Heidelberg (2003)

4. Canetti, R., Krawczyk, H.: Analysis of key-exchange protocols and their use for building secure channels. In: Pfitzmann, B. (ed.) EUROCRYPT 2001. LNCS, vol. 2045, pp. 453-474. Springer, Heidelberg (2001), http://eprint.iacr.org/2001/040

5. Canetti, R., Krawczyk, H.: Security analysis of IKE. In: Yung, M. (ed.) CRYPTO 2002. LNCS, vol. 2442, pp. 143-161. Springer, Heidelberg (2002), http://eprint.iacr.org/2002/120

6. Choo, K., Boyd, C., Hitchcock, Y.: Examining indistinguishability-based proof models for key establishment protocols. In: Roy, B. (ed.) ASIACRYPT 2005. LNCS, vol. 3788, pp. 585-604. Springer, Heidelberg (2005)

7. Diffie, W., van Oorschot, P., Wiener, M.: Authentication and authenticated key exchanges. Designs, Codes and Cryptography 2, 107-125 (1992)

8. Harkins, D., Carrel, D.: The internet key exchange (IKE)., RFC 2409, Internet Engineering Task Force (1998)

9. Kaufman, C. (ed.): Internet key exchange (IKEv2) protocol, RFC 4306, Internet Engineering Task Force (2005)

10. Krawczyk, H.: SIGMA: The 'SIGn-and-MAc' approach to authenticated DiffieHellman and its use in the IKE protocols. In: Boneh, D. (ed.) CRYPTO 2003. LNCS, vol. 2729, pp. 400-425. Springer, Heidelberg (2003)

11. Krawczyk, H.: HMQV: A high-performance secure Diffie-Hellman protocol., Cryptology ePrint Archive, Report 2005/176, http://eprint.iacr.org/2005/176 In: Shoup, V. (ed.) CRYPTO 2005. LNCS, vol. 3621, pp. 546-566. Springer, Heidelberg (2005)

12. Krawczyk, H.: "HMQV in IEEE P1363", submission to the IEEE P1363 working group, July 7 (2006), http://grouper.iee.org/groups/1363/P1363-Reaffirm/ submissions/krawczyk-hmqv-spec.pdf 
13. LaMacchia, B., Lauter, K., Mityagin, A.: Stronger security of authenticated key exchange. In: Susilo, W., Liu, J.K., Mu, Y. (eds.) ProvSec 2007. LNCS, vol. 4784, pp. 1-16. Springer, Heidelberg (2007)

14. Lauter, K., Mityagin, A.: Security analysis of KEA authenticated key exchange. In: Yung, M., Dodis, Y., Kiayias, A., Malkin, T.G. (eds.) PKC 2006. LNCS, vol. 3958, pp. 378-394. Springer, Heidelberg (2006)

15. Law, L., Menezes, A., Qu, M., Solinas, J., Vanstone, S.: An efficient protocol for authenticated key agreement. Designs, Codes and Cryptography 28, 119-134 (2003)

16. Menezes, A., Ustaoglu, B.: Security arguments for the UM key agreement protocol in the NIST SP 800-56A standard. In: Proceedings of ASIACCS 2008, pp. 261-270. ACM Press, New York (2008)

17. Menezes, A., Ustaoglu, B.: Comparing the pre- and post-specified peer models for key agreement, Technical Report CACR 2008-07, University of Waterloo (2008), http://www.cacr.math.uwaterloo.ca

18. Okamoto, T.: Authenticated key exchange and key encapsulation in the standard model. In: Kurosawa, K. (ed.) ASIACRYPT 2007. LNCS, vol. 4833, pp. 474-484. Springer, Heidelberg (2007)

19. SP 800-56A Special Publication 800-56A, Recommendation for Pair-Wise Key Establishment Schemes Using Discrete Logarithm Cryptography, National Institute of Standards and Technology (March 2006)

20. Ustaoglu, B.: Obtaining a secure and efficient key agreement protocol from (H)MQV and NAXOS. Designs, Codes and Cryptography 46, 329-342 (2008) 